\title{
COMPOSITION FACTORS FOR INDECOMPOSABLE MODULES
}

\author{
DIETER HAPPEL
}

\begin{abstract}
Let $k$ be a field and $A$ be a finite-dimensional algebra over $k$ having only a finite number of isomorphism classes of indecomposable $A$-modules. Let $M, N$ be two indecomposable $A$-modules. Then a homomorphism $f: M \rightarrow$ $N$ is said to be irreducible if for every factorization $f=g h, g$ is split mono or $h$ is split epi [2]. The aim of this note is to give an elementary proof of the fact that the indecomposable $A$-modules are completely determined, up to isomorphism, by their composition factors if there is no chain of irreducible maps from an indecomposable module to itself. This theorem was first proved in [5] involving the theory of tilted algebras.
\end{abstract}

By $\bmod A$ we denote the category of finitely generated (right) $A$-modules and ind $A$ denotes the full subcategory of $\bmod A$ consisting of the indecomposable $A$ modules. $A$ is said to be of finite representation type if ind $A$ consists only of a finite number of isomorphism classes of indecomposable modules. Let $S_{1}, \ldots, S_{n}$ be the simple modules and let $P(j), 1 \leq j \leq n$, be the indecomposable projective module with top $P(j)=S_{j}$. Let $M$ be in $\bmod A$. The number of times $S_{j}$ occurs in a composition series of $M$ clearly equals $\operatorname{dim}_{\mathrm{End} P(j)} \operatorname{Hom}(P(j), M)$. This defines a vector $\operatorname{dim} M=(\operatorname{dim} \operatorname{Hom}(P(j), M))_{j}$ usually called the dimension type of $M$. For example consider the following four-dimensional algebra given by

$$
A=\left\{\left(\begin{array}{llll}
a & 0 & 0 & 0 \\
0 & a & 0 & d \\
c & 0 & b & 0 \\
0 & 0 & 0 & b
\end{array}\right), a, b, c, d \in k\right\}
$$

Then $A$ has exactly four indecomposable modules namely $S_{1}, S_{2}, P(1)$ and $P(2)$ and obviously $\underline{\operatorname{dim}} P(1)=\underline{\operatorname{dim}} P(2)$. For the convenience of the reader we will recall some of the basic notions in representation theory [2]. Denote by $A^{\text {op }}$ the opposite algebra and by $D=\operatorname{Hom}(-, k)$ the usual duality from $\bmod A^{\text {op }} \rightarrow \bmod A$. Let $M$ be an indecomposable nonprojective $A$-module. Consider a minimal projective resolution $P^{\prime} \stackrel{\oplus}{\rightarrow} P \stackrel{\pi}{\rightarrow} M \rightarrow 0$ then by definition $\operatorname{Tr} M=\operatorname{Cok}(\operatorname{Hom}(\epsilon, A))$, hence $D \operatorname{Tr} M$ is again an $A$-module. Dually we can define $\operatorname{Tr} D M$ for any indecomposable noninjective $A$-module. A nonsplit exact sequence of $A$-modules $0 \rightarrow X \stackrel{f}{\rightarrow} Y \stackrel{\circ}{\rightarrow} Z \rightarrow$ 0 is called an Auslander-Reiten sequence provided $X$ and $Z$ are indecomposable and satisfying either of the following equivalent conditions:

(a) Given a nonisomorphism $h: M \rightarrow Z$ with $M$ indecomposable, there is a $t: M \rightarrow B$ such that $h=g t$.

(b) Given a nonisomorphism $j: X \rightarrow N$ with $N$ indecomposable, there is an $s: Y \rightarrow N$ such that $j=s f$.

Received by the editors September 1, 1981.

1980 Mathematics Subject Classification. Primary 16A46, 16A64; Secondary 16A53.

(C) 1982 American Mathematical Society 0002-9939/81/0000-0116/801.50 
A nonsplit morphism $X \stackrel{f}{\rightarrow} Y$ with $X$ indecomposable and satisfying (b) is called a left almost split map.

The existence and uniqueness theorem says that given any nonprojective indecomposable module $Z$ there is a unique, up to isomorphism, Auslander-Reiten sequence $0 \rightarrow D \operatorname{Tr} Z \rightarrow Y \rightarrow Z \rightarrow 0$ and, dually, given any indecomposable noninjective module $X$ there is a unique, up to isomorphism, Auslander-Reiten sequence $0 \rightarrow X \rightarrow Y \rightarrow \operatorname{Tr} D X \rightarrow 0$. For $X$ indecomposable there is a unique, up to isomorphism, minimal left almost split map, $X \rightarrow Y$. If $X$ is noninjective then $Y$ is the middle term of the Auslander-Reiten sequence starting with $X$. If $X$ is injective then $Y=X / \operatorname{soc} X$. To $A$ we can associate the Auslander-Reiten quiver $\Gamma(A)$ in the following way: $\Gamma(A)$ has as vertices representatives from the isomorphism classes of indecomposable modules. There is an arrow from a class $M$ to a class $N$ if there is an irreducible map from $M$ to $N$. This is a locally finite graph having in general several connected components. A component $C$ of $\Gamma(A)$ is called preprojective provided $C$ does not contain oriented cycles and all $M \in C$ are of the form $\operatorname{Tr} D^{n} P$ for some $n \in \mathbf{N}$ and some indecomposable projective module $P$ [5]. We will use the following facts for modules in $C$. If $X, Y \in C$ such that $\operatorname{Hom}(X, Y) \neq 0$ then there is a path from $X$ to $Y$. On the other hand if there is no path from $X$ to $Y$ then $\operatorname{Hom}(X, Y)=0$. We allow the trivial path of length zero to be a path.

THEOREM. Let $C$ be a preprojective component of $\Gamma(A)$. Let $M, N$ be in $C$ with $\underline{\operatorname{dim}} M=\underline{\operatorname{dim}} N$ then $M \stackrel{\cong}{\cong}$.

Proof. Let $X$ be in $C$. There is an indecomposable projective $P$ in $C$ and a path from $P$ to $X$. Denote by $c(P, X)$ the maximal length of all such paths. Let $h(X)=\max c(P, X)$ where $P$ runs through all projective modules in $C$ having a path to $X$. Since $C$ does not contain an oriented cycle we have that $h(X)$ is finite for $X \in C$. Observe that for $X$ nonprojective we have $h(X)>0$. Now assume $M$ is not isomorphic to $N$. Consider the left almost split maps $M \rightarrow B$ and $N \rightarrow E$. Let $B=\bigoplus_{i=1}^{r} B_{i}$, with $B_{i}$ indecomposable and $E=\bigoplus_{j=1}^{s} E_{j}$, with $E_{j}$ indecomposable. Consider the following subsets of the vertices of $C$. $\mathcal{Y}_{i}=\{Y \in C \mid$ there is a path from $Y$ to $\left.B_{i}\right\}$ and $Z_{j}=\left\{Z \in C \mid\right.$ there is a path from $Z$ to $\left.E_{j}\right\}$. Let $y=\bigcup_{i=1}^{r} y_{i}$ and $Z=\bigcup_{j=1}^{s} Z_{j}$.

Let $Y^{\prime}=\left\{Y^{\prime} \in C \mid Y^{\prime}=D \operatorname{Tr} Y\right.$ for $\left.Y \in \mathcal{Y}\right\}$ and $Z^{\prime}=\left\{Z^{\prime} \in C \mid Z^{\prime}=D \operatorname{Tr} Z\right.$ for $Z \in Z\}$. Clearly $M \notin Y^{\prime}$ and $N \notin Z^{\prime}$. Without loss of generality we may assume $N \notin y^{\prime}$. Otherwise we would have $N \in y^{\prime}$ and $M \in Z^{\prime}$. This means $\operatorname{Tr} D N \in Y_{i}$ for one $i$ and $\operatorname{Tr} D M \in Z_{j}$ for one $j$, hence there is a path from $\operatorname{Tr} D N$ to $B_{i}$ and a path from $\operatorname{Tr} D M$ to $E_{j}$. But this will give an oriented cycle in $C$ contrary to our assumption. We prove by induction on $h$ that for all $Y \in \mathcal{Y}$ we have $\operatorname{dim} \operatorname{Hom}(Y, M)=\operatorname{dim} \operatorname{Hom}(Y, N)$. Indeed if $h(Y)=0$ then $Y$ is projective and the assertion follows from the fact that $\underline{\operatorname{dim}} M=\underline{\operatorname{dim}} N$. If $h(Y)=n$ then either $Y$ is projective and again the assertion follows from our hypothesis or else $Y$ is not projective. In this case consider the Auslander-Reiten sequence $0 \rightarrow D \operatorname{Tr} Y \rightarrow$ $E \rightarrow Y \rightarrow 0$. Let $E=\bigoplus_{i=1}^{s} E_{i}$ with the $E_{i}$ indecomposable. With $Y \in \mathcal{Y}$ we also have $D \operatorname{Tr} Y, E_{i} \in \mathcal{Y}$ and $h(D \operatorname{Tr} Y)<n$ and $h\left(E_{i}\right)<n$. Now apply the functors $\operatorname{Hom}(-, M)$ and $\operatorname{Hom}(-, N)$ to the above sequences. Since $D \operatorname{Tr} Y \neq$ $M, N$ these functors are exact, hence $\operatorname{dim} \operatorname{Hom}(Y, M)=\operatorname{dim} \operatorname{Hom}(Y, N)$. Now consider again the minimal left almost split map $M \rightarrow B$. Since $M, B_{i} \in \mathcal{Y}$ we have 
$\operatorname{dim} \operatorname{Hom}(M, M)=\operatorname{dim} \operatorname{Hom}(M, N)$ and $\operatorname{dim} \operatorname{Hom}\left(B_{i}, M\right)=\operatorname{dim} \operatorname{Hom}\left(B_{i}, N\right)$. The first equation tells us that $\operatorname{Hom}(M, N) \neq 0$. On the other hand we have $\operatorname{Hom}(B, M)=0$ since $C$ does not contain an oriented cycle thus $\operatorname{Hom}(B, N)=0$. But this gives the desired contradiction. Namely if $f \in \operatorname{Hom}(M, N)$, with $f \neq 0$ we get a nonzero map from $B$ to $N$ by the property of the left almost split map. Hence $M \cong N$.

We have the following immediate application to algebras of finite representation type.

COROLLARY. Let $A$ be of finite representation type and indecomposable as algebra. Suppose $\Gamma(A)$ does not contain an oriented cycle. Then the indecomposable modules are determined, up to isomorphism, by their composition factors.

Proof. If $A$ is indecomposable as algebra then it is well known that $\Gamma(A)$ is connected [1]. Hence $\Gamma(A)$ is a preprojective component.

Examples of classes of algebras of finite representation type without oriented cycles in their Auslander-Reiten quiver may be found in $[\mathbf{3}, \mathbf{4}, \mathbf{5}]$.

\section{REFERENCES}

1. M. Auslander, Functors and morphisms determined by objects, Proc. Conf. on Representation Theory (Philadelphia 1976), Dekker, New York, 1978, pp. 1-244.

2. M. Auslander and I. Reiten, Representation theory of Artin algebras. III, VI, Comm. Algebra 3 (1975), 239-294; 5 (1977), 443-518.

3. R. Bautista and F. Larion, Auslander-Reiten quivers for certain algebras of finite representation type (to appear).

4. K. Bongartz and C. M. Ringel, Representation-finite tree algebras (to appear).

5. D. Happel and C. M. Ringel, Tilted algebras, Trans. Amer. Math. Soc. (to appear).

Department of Mathematics, BRandeis University, Waltham, MassachuSETTS 02254

Current address: Fakultät für Mathematik, Universität Bielefeld 1, West Germany 